Sonia Vaupot* $\quad$ UDK [81'373.4:355.01]:821.133.1Barbusse H.=163.6

Université de Ljubljana

DOI: $10.4312 /$ linguistica.58.1.65-76

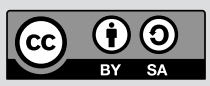

\title{
LA TRADUCTION SLOVÈNE DE L'ARGOT MILITAIRE DANS LE FEU DE BARBUSSE
}

\section{INTRODUCTION}

En 1914, quand la Première Guerre mondiale éclate, malgré une santé fragile, un statut d'auteur déjà bien établi et ses quarante et un ans, Adrien Gustave Henri Barbusse (1873-1935) s'engage volontairement dans l'armée. Il va ainsi combattre dans les tranchées du front. C'est là qu'il tient un carnet de guerre où il note des expériences vécues, des expressions de poilus, et dresse des listes. Il utilisera ce carnet pour rédiger son roman qui connaîtra une critique sévère, car il se présente à la fois comme un roman et comme le journal d'une escouade. Blessé, Barbusse sera réformé après avoir passé 22 mois dans les tranchées (1914-1916). De cette expérience naîtra Le Feu, sous-titré Journal d'une escouade. C'est donc un roman de guerre inspiré par le vécu de son auteur. Il paraît d'abord sous forme de feuilleton, dans le quotidien L'Euvre à partir d'août 1916, puis intégralement à la fin de novembre 1916 aux éditions Flammarion. Il reçoit la même année le Prix Goncourt.

Le récit réaliste, composé de 24 chapitres, dresse un portrait brutal, mais aussi émouvant des atrocités de la guerre et des soldats qui y ont partagé la souffrance. Les lieux du combat se situent dans le Nord-Pas-de Calais. Le roman a été traduit en slovène en 1921 par Anton Debeljak, sous le titre Ogenj (dnevnik desetnije). Anton Debeljak n'était pas seulement traducteur, il était aussi poète, prosateur, essayiste, journaliste et critique littéraire. Cinq années séparent donc la traduction du texte original.

Dans cette contribution, nous poserons d'abord le contexte culturel qui a donné naissance à l'oeuvre de Barbusse, et nous établirons des parallèles entre la Première Guerre mondiale vécue en France et en Slovénie. Notre but sera ensuite de mettre en valeur les difficultés face à la traduction de l'argot militaire issu d'une période bien particulière. Nous commencerons par des données générales sur l'écrivain et le contexte culturel qui a donné naissance à son œuvre. Nous poursuivrons en précisant la présence des termes issus de l'argot militaire dans le roman et, enfin, nous aborderons les procédés adoptés pour traduire l'argot en slovène.

\section{LE CONTEXTE CULTUREL}

L'œuvre de Barbusse est une œuvre engagée qui défend des idées pacifistes, dénonce ceux qui se sont enrichis grâce à la guerre et témoigne des souffrances des soldats durant la Première Guerre mondiale. Le récit réaliste rend aussi hommage aux

sonia.vaupot@ff.uni-lj.si 
camarades que Barbusse a côtoyés. Le texte a donc une valeur littéraire, mais surtout documentaire.

\subsection{La Grande Guerre en France}

Le 28 juin 1914, l'attentat de Sarajevo en Bosnie entraîne l'Europe dans la guerre. L'Allemagne déclare la guerre à la France le 3 août 1914. Dès lors, la France sera plongée dans une guerre de quatre ans qui bouleversera les aspects politiques, économiques et sociaux du pays. L'ordre de mobilisation est lancé et plusieurs millions de soldats français partent au front. La Grande Guerre sera la première guerre totale de l'histoire de France. Elle mènera à une forte mobilisation des soldats, mais aussi à celle de l'économie, de l'agriculture, de l'industrie, des sciences et techniques.

Dès le mois d'août et jusqu'à la fin de 1914, la vie politique est accaparée par les contraintes militaires. La guerre se déroulera sur deux fronts : le front de l'avant (où vivent les soldats et les poilus) et le front intérieur ou l'arrière (la population civile). Mais, une forte interaction entre les deux fronts apparaitra tout au long de la guerre, ce qui bouleversera la vie des Français et débouchera sur un traumatisme. Cette guerre traumatique brutalisera la société, favorisant le développement de la haine et de la violence.

On compte une première phase, en 1914, qui est une phase de mouvement où chaque armée essaie, en se déplaçant, de contourner l'autre armée. Puis, le front se stabilise et la guerre de position commence. En effet, une deuxième phase, de 1915 à 1917, correspondra à la guerre des tranchées, elle sera jalonnée de batailles meurtrières. Les conditions de combats sur le front sont très difficiles et elles ne cessent de se dégrader à mesure que le conflit s'éternise. Jusqu'en 1917, les poilus acceptent la guerre avec patriotisme. Dans les réseaux de tranchées, ils vivent dans des conditions très difficiles. Le nombre de mutilés, « les gueules cassées », est considérable. Face à cette terrible situation, une fraternité se développe entre des hommes d'origine sociale et ethnique différente. À l'arrière, la population est mobilisée par la guerre totale et participe à l'effort de guerre. Les civils doivent ravitailler les soldats et donner pour la Patrie. Quant à l'État, il tente de contrôler les esprits de la population afin de répandre l'idée que tout se passe bien sur le front, par le développement du patriotisme, de la propagande et de la répression face à la montée du pacifisme et aux grèves. 1918 voit la reprise de la guerre de mouvement.

\subsection{La Première Guerre mondiale en Slovénie}

Lorsque la Première Guerre mondiale éclate, la Slovénie fait partie de l'Empire austro-hongrois. Pendant plus de trois ans, les Slovènes vont se battre dans les rangs de l'armée austro-hongroise sur le front de l'Isonzo et d'autres champs de bataille. Durant cette guerre, l'armée royale italienne et ses alliés mènent des opérations militaires contre les armées de l'Autriche-Hongrie et de l'Allemagne dans l'Italie nordorientale. L'Italie espérait obtenir les provinces du Trentin, Trieste et d'autres territoires tels que le Tyrol du Sud, 1'Istrie et la Dalmatie. En 1915, ce pays voisin de l'actuelle Slovénie tente de percer le front qui se stabilise dans la région des vallées 
d'Isonzo, mais tend vers la capitale Ljubljana. La première offensive vise à conquérir la ville de Gorizia au-delà de la rivière Isonzo (en slovène, Soča). Après une première avancée italienne, les Austro-Hongrois résistent. La guerre devient alors une guerre de tranchées semblable à celle que connait notamment la France. Mais, sur le front italien, ces tranchées ne sont pas creusées dans la terre, elles sont sculptées dans la roche des Alpes, donc dans les montagnes.

Plusieurs attaques auront lieu. Les batailles de l'Isonzo sont marquées par douze affrontements violents entre l'Italie et l'Autriche-Hongrie qui se sont déroulés entre juin 1915 et septembre 1917. Celles qui ont le plus touché la Slovénie se sont déroulées de mai 1917 à novembre 1917. La 10 ${ }^{\mathrm{e}}$ attaque avait notamment pour objectif de briser le front austro-hongrois pour accéder à la ville de Trieste. La $11^{\mathrm{e}}$ attaque touchera la ville de Gorizia et la dernière bataille correspond à celle de Caporetto.

Deux œuvres littéraires racontent cette bataille. Comme Henri Barbusse, Hemingway et Prežihov Voranc vont s'inspirer de leur propre expérience pour construire leurs romans. Le roman de guerre L'adieu aux armes (1929) d'Ernest Hemingway qui mêle une histoire d'amour romantique et le récit des batailles qui se déroulent sur le front, là où les Italiens se battent contre les Austro-Hongrois, à l'est d'Udine, près de la ville de Gorizia. Hemingway plante la seconde partie de son roman au milieu de la déroute de Caporetto, lors des attaques qui ont lieu en octobre et novembre 1917. Les Austro-Hongrois, appuyés par des troupes allemandes, enfoncent le front dans la vallée de l'Isonzo, provoquant la débâcle de l'armée italienne. L'auteur s'était, par ailleurs, lui-même engagé dans les ambulances de la Croix rouge en Italie, sur le front de l'Isonzo, pendant la Première Guerre mondiale où il avait été blessé.

Un autre roman de guerre autobiographique Doberdob (1940), du romancier slovène Prežihov Voranc, raconte notamment les combats qui se sont déroulés sur le front de l'Isonzo. Doberdò del lago est une commune de la province de Gorizia située dans la région du Frioul-Vénétie. L'auteur combat dans les rangs de l'armée austro-hongroise sur le front de l'Isonzo et raconte son vécu jusqu'à la débâcle italienne. Après la chute de la monarchie austro-hongroise en 1918, l'État des Slovènes, Croates et Serbes est fondé pour une courte période, avant de se réunir dans le Royaume des Serbes, des Croates et des Slovènes.

Par conséquent, la Première Guerre mondiale frappe durement la Slovénie. On compte, comme en France, de nombreuses pertes dans l'arrière-pays et sur les fronts qui traversaient une partie du territoire slovène. Bien que combattant en altitude, les soldats slovènes ont connu une guerre de tranchées semblable à celle du front occidental. On en déduit qu'il devait exister aussi un argot militaire slovène.

\section{LE VOCABULAIRE ARGOTIQUE}

La première définition de l'argot est une définition historique. D'après le Trésor de la langue française, l'argot était la langue des malfaiteurs. Il désignait la langue de l'ensemble des gueux, bohémiens, mendiants professionnels et voleurs. Au cours des siècles, les acceptions se sont multipliées. On parle d'ailleurs souvent d'argots (au 
pluriel) ou de «parlures argotiques », pour reprendre l'expression de Denise FrançoisGeiger et Jean-Pierre Goudaillier (1991).

Plusieurs définitions ont été élaborées notamment au cours du $\mathrm{XX}^{\mathrm{e}}$ siècle. Pour Guiraud, l'argot est défini comme un signum linguistique, « un signum de classe, de caste, de corps » (1956: 97), une certaine façon de parler par laquelle l'individu et le groupe se distinguent (ibid. : 102). L'argot est également devenu une « langue refuge, emblématique, la langue des exclus, des marginaux ou de ceux qui se veulent tels, en même temps qu'une façon pour certains de marquer leur différence par un clin d'œil linguistique » (Calvet 1994 : 9). Le Dictionnaire de linguistique désigne l'argot comme « un dialecte social réduit au lexique de caractère parasite (dans la mesure où il ne fait que doubler, avec des valeurs affectives différentes, un vocabulaire existant), employé dans une couche déterminée de la société qui se veut en opposition avec les autres » (Dubois et al. $2002:$ 48).

L'argot est ainsi un langage ou un vocabulaire particulier qui se crée à l'intérieur de groupes déterminés et par lequel l'individu affiche son appartenance au groupe. C'est notamment un phénomène lexical, mais aussi une ressource stylistique.

\subsection{Le lexique argotique des tranchées}

À la parution du roman d'Henri Barbusse, les avis sont partagés. Certains vont déplorer le langage dans lequel s'expriment les personnages du Feu. Ainsi, Cru (1929 : 13) observera que «Barbusse a visiblement noté sur un carnet toutes ces diverses formes qu'un farceur lui a fait prendre pour de l'argot en usage, puis il les a entassées dans son roman, attribuant à deux ou trois soldats les gros mots cueillis sur les lèvres d'un très grand nombre d'individus. » Au contraire, Spitzer (1920:59) estime que ce roman témoigne d'une « conception scientifiquement parfaite de l'argot des tranchées. »

Après la mort de Barbusse, les lettres qu'il a écrites à sa femme de 1914 à 1917 sont publiées. Ces lettres témoignent de la fascination de l'auteur pour l'argot populaire. Il y évoque la documentation écrite qu'il a amassée pour le roman (1937: 192) : « [...] je bâtis actuellement tout le bouquin, et c'est justement le moment où rien ne doit manquer de ma documentation. » Il ajoute que cette documentation est composée « [...] en grande partie d'expressions pittoresques trop abondantes pour rester dans ma mémoire à la portée de ma plume, si je puis dire, il me faut des notes écrites ».

$\mathrm{Si}$ les termes argotiques et techniques détiennent une place importante dans l'œuvre de Barbusse, l'argot y est spécifique puisqu'il s'agit du parler militaire, plus particulièrement de l'argot des poilus et des tranchées. Le terme de « poilu » qualifie les soldats français au cours de la guerre de 1914-1918. On pense souvent à tort que ce mot désignait des soldats des tranchées qui étaient caractérisés par une forte pilosité. Privés de condition d'hygiène convenable, les poilus se laissaient pousser barbes et cheveux. Or ce terme est attesté dès le XIX ${ }^{\mathrm{e}}$ siècle pour désigner un soldat endurant et courageux. En effet, dans l'argot militaire, le poil est un signe de virilité, de courage ou d'expérience. C'était donc bien pour signifier le courage, la force et la virilité qu'on a qualifié les soldats français de poilus. 
L'argot militaire existait déjà bien avant la Grande Guerre, il a ensuite été repris et transformé durant le conflit. Pour Déchelette (1918), les poilus avaient besoin d'un vocabulaire spécifique qui les distinguait de la population civile de «l'arrière » et asseyait leur identité de groupe pour mieux défendre leurs intérêts : « (...) le poilu créa des mots qui désignaient ces choses nouvelles ou faisaient mieux image que les mots de la langue courante ; il donna de nouvelles significations à des mots d'argot ancien ou moderne, de patois provinciaux ou de français » (Ibid.: 5). On retrouve, dans l'œuvre de Barbusse, des termes simples et répandus, des termes régionaux, et au moins deux niveaux de langue : une langue orale et un niveau plus soutenu, plus littéraire. Du reste, l'auteur en convient : "Le même parler fait d'un mélange d'argots d'atelier et de caserne et de patois, assaisonné de quelques néologismes, nous amalgame, comme une sauce, à la multitude compacte d'hommes qui, depuis des saisons, vide la France pour s'accumuler au Nord-Est » (Barbusse 1916 : 17). Dauzat (1918) confirme que cet argot n'était pas une langue née de la guerre. Il était alimenté par l'ancien argot de caserne, avec lequel les mobilisés avaient renoué, et le langage populaire de l'ouvrier. Il écrira (1917 : 655) « D'ailleurs l'argot militaire est essentiellement un langage parlé : tout ce qui se dit ne s'écrit pas. Les nombreuses missives de soldats (appartenant à diverses classes sociales), dont nous avons eu les originaux entre les mains, ne contenaient presque aucune trace d'argot militaire.»

Pour notre part, nous nous contenterons de proposer un classement sommaire des termes argotiques employés par Barbusse. À cet effet, nous nous sommes basées sur la classification proposée par Déchelette (1918: 8-14) dans son ouvrage. Nous nous sommes limitées à un maximum de dix termes ou expressions pour chacune des catégories présentées ci-dessous. Ces termes ou expressions sont susceptibles de poser des problèmes de traduction, du fait de leur non-appartenance à la langue orale courante. Ainsi, nous relevons, dans le roman de Barbusse, d'un point de vue thématique, lexical et stylistique :

- Des mots de technique militaire (armes) : mitrailleur, mitrailleuse, lebel, mauser, la baïonnette Rosalie, seringue, marmites, cure-dents, boîte de singe, voltigeur, etc.

- Des mots d'argots militaires spéciaux (aviation, automobilisme) : chasseur, manche à balai, bagnole, Rosalie, taube, aéro, vaguemestre, etc.

- Des mots de caserne : rabiot, jus, adjupette (pour adjudant), doublard ou capiston (pour capitaine), pajot ou fayot (pour la nourriture), caberlot (pour la tête), barda ou bardin (pour le bagage du soldat), etc.

- Des mots qui sont intimement liés à la vie du poilu : réseau, voltigeur, relève, artillerie, musette, et les dérivés de boche comme fantaboches, les feuillées (pour les toilettes des tranchées), etc.

- Des mots d'argot parisien appliqués à la guerre : zigouiller, louper, poteau, boulotter, le pucier (pour le lit) ; avoir les foies ; en lousdoc (insidieusement), se mettre la tringle (être privé de quelque chose), le brisque, etc.

- De vieux mots de français ou de patois provinciaux : marre, marrer, bourrin, naz, etc. En outre, la langue du Nord transparaît grâce au personnage de Bécuwe qui introduit des métaplasmes (bric'ker, n'affaire pour une affaire, l'marmite, 
s'pâtée, etc) et des faits de prononciation particuliers comme la prééminence du -i (vir pour voir, minge pour mange, ine grille », etc.) ou la transformation des chuintantes en vélaires sourdes $[\mathrm{k}]$ : cauffer, kien, kemise, etc.

- Des mots provenant des troupes coloniales (algériennes) : guitoune

- Des mots étrangers, allemands, anglais ou italiens : schlass, shrapnell, bath, palace, etc.

- Des mots imagés pour désigner des choses nouvelles ou créer un doublet argotique: cagoule, museau, etc.

- Des mots français auxquels on a donné un sens nouveau ou que l'on a déformés : graisse d'hérisson, brosse à graisse, guetteur, taper sur l'os, embusquer, moulin à café, etc.

- Les déformations par apocope, aphérèse ou par application de suffixe argotique : pitaine, juteux, colon, perme, tringlot, etc. Parmi ces figures, on note aussi des apocopes (s'mett' à quatt' pattes, I'm' faut mes péniches), des syncopes (f'sais, p'têt' bien) et des aphérèses (mon ieux, l'pitaine), etc.

- Les figures de mots et métonymie : queue de billard, peau de mouche, marmite, nez de rat, boyau, etc.

\section{LES LIMITES DE LA TRADUCTION}

La création littéraire française s'est souvent nourrie de l'argot (voir Cellard 1985). Pourtant, on a tendance à considérer l'œuvre de Barbusse comme un document plutôt qu'une véritable création littéraire. En effet, l'usage de l'argot en littérature n'était pas nouveau, on le voyait comme artificiel et nuisible à la langue française. Pour sa part, Barbusse utilise l'argot comme une langue d'écriture, celle des tranchées, qu'il oppose à la langue officielle de l'écrivain.

La traduction de l'argot pose un certain nombre de difficultés. En effet, dans deux langues différentes, la diversité des sociolectes n'est pas la même : le slovène ne connaît pas l'exact équivalent de ce qu'est l'argot français. La traduction de l'argot technique ou militaire n'est pas une exception. A guise d'exemple, le sous-titre de l'œuvre de Barbusse « Journal d'une escouade » pose un premier problème de traduction : « l'escouade » est la plus petite unité de l'armée française, elle regroupe en théorie 15 soldats sous le commandement d'un caporal. Or l'équivalent slovène « desetnija » correspond à une unité militaire qui regroupe 10 soldats. La réalité n'est donc pas la même.

« L'Argot tend à être un instrument de mystère » (Niceforo 1912:29) : il est vrai que le langage des militaires est affecté d'un marquage implicite, difficile à comprendre dans un autre contexte culturel et à traduire. Le traducteur étant tenu de connaître les écarts entre deux langues de même que les similitudes, les points distinctifs ou communs de l'argot dans deux langues sont des éléments clés offrant des issues dans la recherche de solutions. Au niveau de la création du lexique argotique, les langues française et slovène partagent de nombreux mécanismes de formation lexicale, quoique les systèmes ne soient pas toujours identiques ou symétriques. Toutefois, ni la réalité ni la productivité lexicale ne sont identiques dans les deux langues et elles fluctuent en fonction de certains éléments comme l'époque, les groupes sociaux, etc. Face à la difficulté 
de la tâche et aux limites de la traduction, plusieurs questions se posent. Le traducteur est-il tenu de respecter l'équivalence lexicale ou stylistique du texte source ? Doit-il avoir recours aux notes du traducteur pour faire passer le message ou, au contraire, préférer d'adapter l'œuvre pour la rendre accessible au lecteur? Enfin, l'argot français a-t-il été traduit par l'argot militaire slovène ? Sans être exhaustive, notre recherche a permis de répertorier certaines stratégies de traduction.

\subsection{Les pertes inévitables}

D'une manière générale, l'usage de la langue orale est respecté dans la traduction slovène : par exemple, la phrase « le pot de chambre te protège suffisamment l'caberlot contre les billes de plomb. (p. 197-198) est traduit par « Ponočna posoda ti zadostno čuva tikvo pred svinčenkami. » (p. 201), mis à la place de «tkivo » (fr. le tissu) ou " glavo » (fr. la tête) en slovène standard. Mais la traduction est parfois rendue dans un registre plus littéraire : ainsi « On vous a oubliés, pauvres vieux!» (p. 50) est traduit par « Pozabili so vaju, ubožca » (p.54). Par ailleurs, le traducteur omet souvent de traduire le terme « mon vieux » tout au long du roman.

Contrairement à l'oeuvre originale, l'ouvrage slovène ne possède pas de préface. On note également un manque de parallélisme, voire des pertes lexicales ou sémantiques entre l'argot français et slovène. Par exemple, le mot « barda » apparaît à plusieurs reprises dans le texte. Dans l'argot des combattants, ce mot désigne l'équipement du soldat. Le terme prend souvent une connotation négative en raison du poids de celuici, qui peut dépasser les $35 \mathrm{~kg}$, et des efforts qu'on doit déployer pour s'équiper ou se déséquiper dans les tranchées. Le terme " barda ", que l'on retrouve notamment dans le titre du chapitre XIV, est traduit en slovène par «breme » tandis que, dans le texte, il est traduit par « prtljaga » :

«Il y a, pour compléter le barda, ce qu'on porte sur son dos. » (p. 168)

«K popolni prtljagi spada še to, kar se nosi na plečih. » (p. 173)

Cette dernière traduction marque une semi-perte sémantique. En effet, c'est le contexte qui nous permet de conclure implicitement à la pénibilité du fardeau.

Par ailleurs, l'emploi d'un archaïsme mène nécessairement à une perte. La phrase suivante « Si, dit le vaguemestre. . est traduite en slovène par « Pač, pravi vozataj. » Le vaguemestre est le militaire chargé de la distribution du courrier aux armées. Son arrivée est guettée par les combattants qui attendent les lettres et colis constituant leur lien avec l'arrière. Le terme « vozataj » est un archaïsme mis pour le conducteur « voznik ou prevoznik ».

La perte est également inévitable lorsqu'une langue ne connait pas l'équivalent exact d'un terme. Dans la phrase suivante, l'expression « manche à balai » est tirée du domaine de l'aviation: " Moi, j'crois plutôt que ce soit, tout là-haut, un client qui s'paye le coup d'œil sur son manche à balai (...) » (p. 199). Il s'agit ici du levier de commande du gouvernail de profondeur et du gauchissement dans beaucoup d'aéroplanes. Le traducteur slovène a repris l'expression imagée « du manche à balai » dans « Jaz pa bi verjel preje, da visoko tam gori kakšen svetec jaše metlo ter ogleduje vso reč (...)» (p. 202). Toutefois, le message est différent. 


\section{2. L'emprunt et la note en bas de page}

Lorsque l'équivalent n'existe pas ou le terme étranger est compréhensible, le traducteur opte parfois pour un emprunt ou une note en bas de page. Une seule note du traducteur apparaît à propos de l'unique phrase mise en italique (p. 308) : la phrase « Nous levons les bras, dit-il » demeure en français dans le texte slovène, mais sa traduction « Udamo se » (p. 313) a été ajoutée dans une note en bas de page.

Le traducteur a aussi opté pour l'emprunt lexical dans certains cas. Par exemple, le terme « poilu » n'est pas traduit en slovène. Il s'agit là d'un terme non traduisible, car il ne possède pas d'équivalent slovène. Il en est de même pour le terme « voltigeur » qui désigne un fantassin non spécialisé, comme le sont les grenadiers et les fusiliers mitrailleurs : «Une vieille les reçoit pour rien, rapport à c'que son vieux, qu'est mort y a cinquante ans, a été voltigeur dans l'temps. » (p. 69). Le voltigeur va à l'attaque avec son fusil et marche dans la $2^{\mathrm{e}}$ vague d'assaut. Le traducteur a certes utilisé l'emprunt « voltižêr », mais ce mot n'apparaît pas dans le dictionnaire slovène SSKJ : « Starka jih je vzela pod streho zastonj, zato ker je bil njen stari, ki je gagnil že pred petdeset leti, svoje dni voltižêr. » (p. 73). Il s'agit ici d'un archaïsme employé dans la langue croate au XIX ${ }^{\mathrm{e}}$ siècle : «Voltižer 3 . hrvatske provizorne pukovnije » et sans doute d'un terme employé dans le jargon militaire slovène à cette époque.

Dans la phrase «Y en a qui le croient au Trésor. » (p. 36), le terme « Trésor » désigne le service du Trésor, mais le sens est ambigu. Par l'emprunt « Nekateri verjamejo o Trésoru. » (p. 42), le traducteur aura peut-être souhaité garder l'ambiguitté ? Plus loin, ce même terme « (...) qui dirige les Trésors et Postes (...)» (p. 90) est en effet traduit par « (...) ki vodi blagajne in poste (...)» (p. 94).

Enfin, l'emprunt « aéro » dans la phrase « (...) i'voulait faire croire qu'y aurait danger d'bombardement d'aéro...» (p. 112) est traduit par « (...) oni pikec je hotel natvesti, da je opasno zastran aero... » (p. 116). La traduction met ici l'accent sur le dialogue et l'oralité de la langue, même si le sens de l'emprunt reste obscur en slovène. Par ailleurs, la suppression de l'élément argotique s'impose parfois, faute d'équivalent. Nous reprenons le terme « aéro », cité précédemment, qui est pourtant traduit dans la phrase suivante « (...) inspecte l'espace en quête d'un aéro. » (p. 199) par l'équivalent slovène non argotique « letalo» dans « (...) voha po vzduhu za letalo. » (p. 202). Ce choix est sans doute stylistique, le contexte ne permettant pas de comprendre l'allusion. Le traducteur distingue, de la sorte, le langage oral du langage écrit, mais le choix des procédés de traduction facilite surtout la compréhension de la phrase ou du texte.

\subsection{Le calque}

Le traducteur opte parfois pour la traduction littérale de l'unité lexicale. On retrouve ce procédé dans la traduction de la phrase « (...) des jardins d'acclimatation de brisquards » (p. 111), ce qui donne en slovène : « (...) ondukaj imaš zbirke, zverinjake našitkarjev » (p. 115). Le « brisquard » correspond à une personne qui possède de nombreuses brisques, c'est-à-dire des insignes en forme de $\mathrm{V}$ renversé (des chevrons) que les poilus portaient sur les manches : on portait à gauche les brisques de présence aux Armées, à droite les brisques de blessures de guerre. Pour la présence aux Armées, 
la première année donnait droit à un chevron, et ensuite chaque semestre à un nouveau chevron. Le terme slovène " našitkar » n'apparaît pas dans le dictionnaire slovène SSKJ, mais uniquement le terme «našitek », l'équivalent de « chevron». Nous sommes ici en présence d'un néologisme ou bien d'un terme issu du jargon militaire slovène.

\subsection{La description et l'explicitation}

On remplace parfois l'élément argotique par la description de sa forme ou sa fonction. Ainsi, la phrase suivante « (...) on est aussi obscurément entassés que dans une guitoune » (p. 69) est traduite par « (...) smo tako trenutno nakopičeni kakor v strelni jami. » (p. 73). La guitoune, en arabe, signifie la tente ou l'abri. C'est plus particulièrement un trou couvert de rondins, de tôles ondulées ou de sacs à terre, où l'on se met à l'abri des obus. La sécurité y est relative et provisoire. La traduction slovène est ici explicite et décrit bien l'abri.

Parfois une traduction requiert une explication. Le traducteur ajoute ainsi des précisions qui ne sont pas formulées dans le texte d'origine. Pour l'exemple suivant, «L'adjudant commandant le détachement de territoriaux qui fait les corvées au Q.G. du C.A. » (p. 36), Barbusse emploie des abréviations (Q.G. du C.A) pour désigner le « quartier général du corps d'armée ». Le traducteur choisit de les expliciter : "Pribočnik in poveljnik domobranskega oddelka, ki oskrbuje upravo v glavnem stanu armadnega zbora. » (p. 41).

\subsection{L'adaptation et la particularisation}

D'une manière générale, le traducteur a choisi de traduire et non pas d'adapter le texte de Barbusse, même s'il a parfois recourt au procédé d'adaptation. Dans la phrase indiquée ci-dessus, le traducteur renforce les propos en ajoutant une connotation culturelle. Cette adaptation donne plus de force à la traduction et l'explicite en partie. Ainsi, « l'adjudant commandant le détachement de territoriaux » devient " pribočnik in poveljnik domobranskega oddelka ». La traduction se réfère ici à un trait culturel spécifique puisque les «Domobranci » étaient des membres de la Garde nationale slovène. On note également un cas de particularisation qui marque le passage d'un élément à portée générale vers un choix concret.

Un autre cas d'adaptation apparait dans la phrase « Des fayots à l'huile, de la dure, bouillie, et du jus. » (p. 21) qui désigne des haricots (fayots), de la terre (la dure) et du café (le jus). Or la traduction « Štorovec na olju, kuhana žilavka, pa brozga » (p. 26) désigne de jeunes pousses qui apparaissent sur la souche d'un arbre (sl. štorovec) et une boisson qui a mauvais goût (sl. brozga). Quant à « la dure », la traduction est certes littérale, mais peu claire en slovène, ce terme pouvant aussi désigner une variété de vigne qui pousse notamment en Herzégovine, voire même une sorte de fouet.

\subsection{La modulation}

L'exemple suivant marque un changement de point de vue par rapport à l'original : « (...) t'as là-d'dans des collections, des jardins d'acclimatation de brisquards » (p. 111). Les poilus sont vus par les journalistes comme des animaux sauvages. L'image a 
été traduite par « la cage à fauve » dans la phrase « (...) ondukaj imaš zbirke, zverinjake našitkarjev. » (p. 115). Le changement peut ainsi être lexical ou structural.

\section{CONCLUSION}

Ce bref panorama nous permet de dresser un bilan partiel du lexique de guerre décrit dans l'œuvre de Barbusse et nous donne une idée de la complexité des problèmes que pose la traduction de l'argot militaire. La Première Guerre mondiale a été le moteur propulseur d'une véritable révolution langagière : son rôle dans l'enrichissement du vocabulaire est indéniable grâce aux apports divers (des langues régionales, des colonies, des langues étrangères, des technicismes, etc.). Certains termes de l'argot de guerre ont disparu au fil des années. De plus, une traduction datant du début du $\mathrm{XX}^{\mathrm{e}}$ siècle peut apparaître actuellement vieillie ou démodée.

Nous avons vu, d'une part, que le traducteur neutralise l'argot en passant parfois de la langue familière à la langue slovène standard ou même littéraire. D'autres fois, il emploie un niveau de langue supérieur. Tous ces changements de niveau de langue impliquent des pertes plus ou moins importantes. Mais malgré des pertes au niveau de la variation linguistique, le traducteur a su trouver le bon ton et parvient à transmettre parfaitement le style de Barbusse. La traduction de l'œuvre de Barbusse prouve que, malgré les pertes, l'argot et les effets de style sont bien traduisibles, mais souvent à des degrés différents.

\section{Bibliographie}

BARBUSSE, Henry (1916) Le Feu (Journal d'une escouade). Paris: Éditions Flammarion. BARBUSSE, Henry (1921) Ogenj: dnevnik desetnije. Trad. Anton Debeljak. Ljubljana : Zal. Tiskovna zadruga.

BARBUSSE, Henri (1937) Lettres à sa femme 1914-1917. Paris : Flammarion.

CALVET, Louis-Jean (1994) L'argot. Paris: PUF. [Que sais-je ?].

CELLARD, Jacques (1985) Anthologie de la littérature argotique des origines à nos jours. Paris: Mazarine.

CRU, Jean Norton (1929) Témoins. Essai d'analyse et de critique des souvenirs de combattants édités en français de 1915 à 1928. Paris : Les Étincelles.

DAUZAT, Albert (1918) L'Argot de la guerre, d'après une enquête auprès des officiers et soldats. Paris: A. Colin.

DAUZAT, Albert (1917) «L'argot militaire pendant la guerre. » Mercure de France 16/04/1917, 655-668.

DECHELETTE, François (1918) L'argot des poilus. Dictionnaire humoristique et philologique. Paris: Les Éditions de Paris.

DUBOIS, Jean et al. (2002) Dictionnaire de la langue française. Paris: Larousse.

GUIRAUD, Pierre (1956) L'argot. Paris: PUF. [Que sais-je ?].

NICEFORO, Alfredo (1912) Le Génie de l'Argot. Paris: Mercure de France.

SPITZER, Leo (1920) Die Sprache der Soldaten. Studien zu Henri Barbusse. Bonn: F. Cohen.

FRANCOIS-GEIGER, Denise/Jean-Pierre GOUDAILLIER (éds.) (1991) Parlures argotiques. Langue française $\mathrm{n}^{\circ} 90$. 
Le Feu, sous-titré Journal d'une escouade est un roman de guerre inspiré par le vécu de son auteur, Adrien Gustave Henri Barbusse. Il paraît intégralement à la fin de novembre 1916 aux éditions Flammarion. Il reçoit la même année le Prix Goncourt. Ce roman a été traduit en slovène en 1921 par Anton Debeljak, sous le titre Ogenj (dnevnik desetnije).

Dans cette contribution, nous nous demanderons s'il existe des parallèles culturels entre la Première Guerre mondiale vécue en France et en Slovénie. Notre but sera également de mettre en valeur les difficultés face à la traduction de l'argot militaire. Nous commencerons par des données générales sur l'écrivain, puis le contexte historique et culturel qui ont donné naissance à son œuvre. Nous poursuivrons en précisant la présence des termes issus de l'argot militaire dans le roman et, enfin, nous aborderons les procédés adoptés pour traduire l'argot en slovène.

Mots-clés : traduction, argot militaire, équivalence culturelle, Première Guerre mondiale

\section{Abstract \\ SLOVENE TRANSLATION OF MILITARY SLANG IN BARBUSSE'S LE FEU (UNDER FIRE)}

Le Feu, subtitled Journal d'une escouade, is a war novel inspired by the experiences of the author, Adrien Gustave Henri Barbusse. It was published at the end of November 1916 by Flammarion Publishing Group. The same year, the novel received the Goncourt Prize. It was translated into Slovene in 1921 by Anton Debeljak, under the title Ogenj (dnevnik desetnije).

Firstly, the paper endeavours to investigate cultural parallels between World War I in France and Slovenia. Then, we highlight the difficulties in translating military slang. We begin with general information on the writer and continue with the historical and cultural context that gave rise to his work. We also suggest a classification of military slang terms and the procedures adopted to translate it into Slovene.

Keywords: translation, military slang terms, cultural equivalence, World War I 


\section{Povzetek \\ PREVAJANJE VOJAŠKEGA SLENGA V SLOVENŠČINO V BARBUSSOVEM ROMANU OGENJ}

Le Feu, podnaslovljen Journal d'une escouade, avtorja Adriena Gustava Henrija Barbussa je vojni roman, ki ga navdihuje avtorjeva izkušnja. Izšel je v celoti konec novembra 1916 pri založbi Flammarion. Istega leta je roman prejel Goncourtovo nagrado. Anton Debeljak je roman prevedel leta 1921 pod naslovom Ogenj (dnevnik desetnije).

$\mathrm{V}$ tem prispevku se najprej vprašujemo, ali obstajajo kulturne vzporednice med prvo svetovno vojno v Franciji in Sloveniji. Želimo tudi poudariti težave, ki lahko nastajajo pri prevajanju vojaškega slenga. Na začetku predstavimo splošne podatke o pisatelju in o zgodovinskem in kulturnem kontekstu, ki je spodbudil njegovo delo. V nadaljevanju obravnavamo vojaški sleng v romanu, predlagamo klasifikacijo in razpravljamo o postopkih, ki jih je prevajalec uporabil pri prevajanju vojaškega slenga v slovenščino.

Ključne besede: prevod, vojaški sleng, kulturna ekvivalenca, prva svetovna vojna 